\title{
Time Resolved Magnetophotoluminescence of Biased GaAs/AlGaAs Double Quantum Well Structure
}

\author{
K. Surowiecka ${ }^{a, *}$, A. Wysmolek ${ }^{a}$, R. Stȩpniewski ${ }^{a}$, \\ M. Potemski ${ }^{b}$ And M. Henini ${ }^{c}$ \\ ${ }^{a}$ Institute of Experimental Physics, University of Warsaw \\ Hoża 69, 00-681 Warsaw, Poland \\ ${ }^{b}$ Grenoble High Magnetic Fields Laboratory, CNRS, Grenoble, France \\ ${ }^{c}$ School of Physics and Astronomy, University of Nottingham, England \\ Time resolved photoluminescence of double quantum well structure was \\ investigated versus electric and magnetic fields applied across the sample. \\ The emission due to direct excitons (electron and hole are localized within \\ the same quantum well) decays fast at the nanosecond timescale, whereas the \\ recombination kinetics of indirect excitons is much slower and spreads over \\ microseconds. The time evolution of indirect exciton emission is shown to be \\ altered by application of either electric or magnetic field. This reflects the \\ non-trivial effects of exciton localization which leads to the non- exponential \\ decays of the indirect exciton emission.
}

PACS numbers: 71.35.Ji, 78.67.De

\section{Introduction}

Biased, double quantum well structures are among most promising systems to attain one of the longstanding challenges of the semiconductor physics: a demonstration of microscopically coherent state of excitonic gas, the Bose-Einstein condensation $[1,2]$. In reference to this well known but usually appearing under thermodynamic equilibrium phenomenon, the finite lifetime of excitonic quasi-particles is the relevant issue. The detailed studies of the kinetics of optical excitations in double quantum well structures, which are presented in this work, are necessary before approaching further, more ambitious tasks.

*corresponding author; e-mail: ksurow@fuw.edu.pl 


\section{Samples and experiment}

The $p^{+}-i-n^{+}$structure was grown by molecular beam epitaxy (MBE) on $n^{+}$-type GaAs:Si $\left(2 \times 10^{18} \mathrm{~cm}^{-3}\right)$. The single pair of $6 \mathrm{~nm}$ GaAs quantum wells separated by the $\mathrm{Al}_{0.33} \mathrm{Ga}_{0.67} \mathrm{As}$ barrier was embedded in the intrinsic part of $300 \mathrm{~nm} \mathrm{Al} \mathrm{Al}_{0.33} \mathrm{Ga}_{0.67} \mathrm{As}$ insulating part of the sample. The top contact layer was $p^{+} 200 \mathrm{~nm}$ GaAs:Be $\left(2 \times 10^{18} \mathrm{~cm}^{-3}\right)$. The separation between wells is $5 \mathrm{~nm}$.

The time resolved photoluminescence in electric and magnetic field was investigated. The energy of the laser line excitation (both continuous wave and pulsed) exceeded the gap of $\mathrm{Al}_{0.33} \mathrm{Ga}_{0.67}$ As. All measurements were performed in liquid helium temperature in the Faraday configuration. The external bias applied to the sample allows changing electric field in the structure, in the direction perpendicular to the plane of the quantum wells. The bias was applied both in forward and reversed direction. The forward bias reduces the external electric field because the external bias compensates the built-in electric field. The reversed bias enhances the electric field in the structure.

\section{Results}

A typical photoluminescence (PL) spectrum is presented in Fig. 1. Two emission lines are observed. The one at the higher energy (about $1.57 \mathrm{eV}$ ) is attributed to the recombination within the same quantum well (direct) while the lower energy line (about $1.153 \mathrm{eV}$ ) originates from the recombination of the indirect excitons, when electron and hole are localized in the neighboring quantum wells. The direct luminescence line consists of two lines which are associated with the recombination of neutral and charged exciton, respectively.

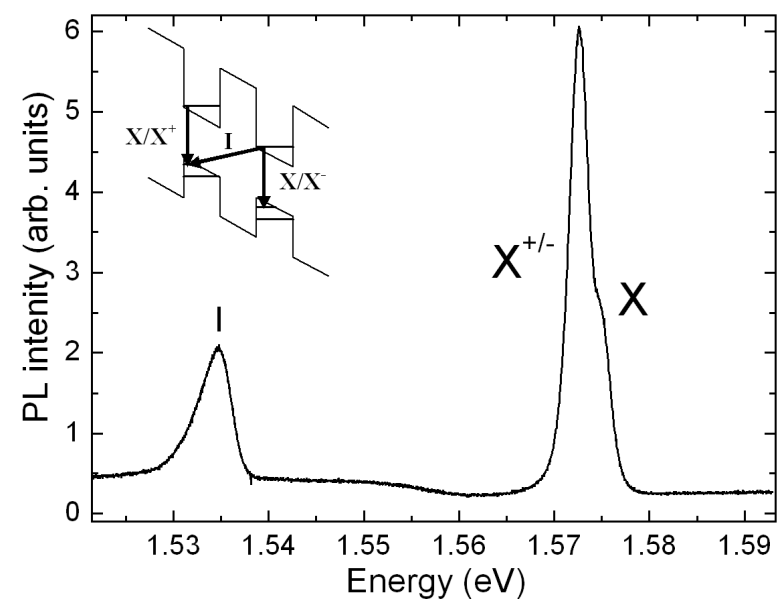

Fig. 1. Representative photoluminescence spectrum of the investigated structure. Inset shows schematic bands profile of the double quantum well system in electric field. Direct $\left(\mathrm{X}, \mathrm{X}^{ \pm}\right)$and indirect $(\mathrm{I})$ transitions are marked with arrows. 


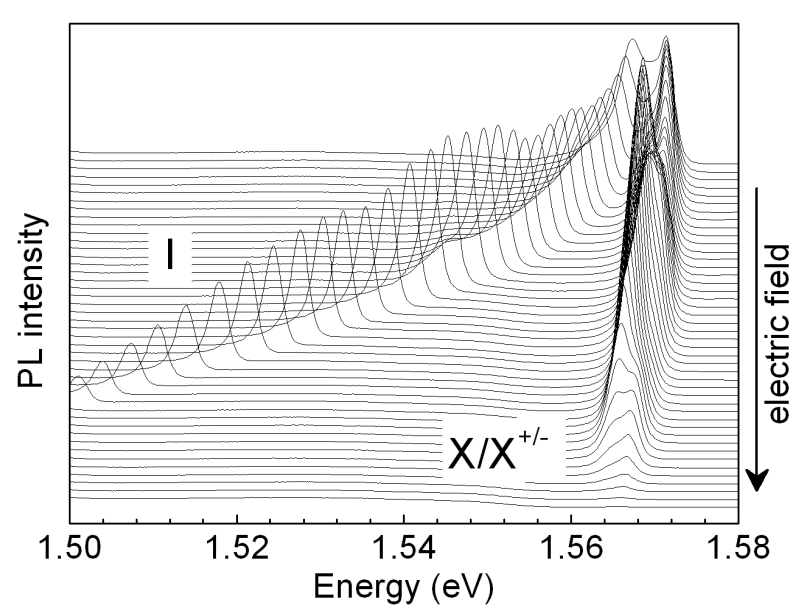

Fig. 2. The evolution of the photoluminescence spectrum under the applied electric field.

The characteristic evolution of the PL spectrum of the structure under the applied electric field is presented in Fig. 2. Increasing the electric field in the structure causes the strong redshift of indirect transitions. At the same time, only small redshift of the direct emission, due to the quantum confined Stark effect, is observed. Hence, the energy position of the indirect exciton can be used as a measure of the electric field in the structure.

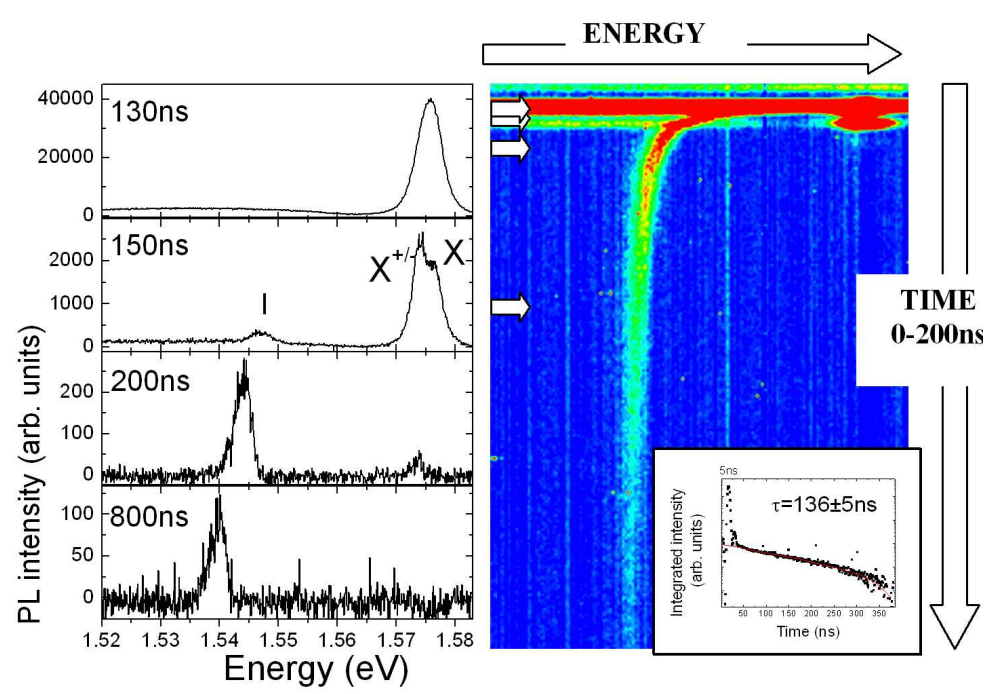

Fig. 3. Temporal evolution of the emission from the structure after the pulsed excitation. The left part shows the photoluminescence collected with different delays after the laser pulse. The right part shows all collected results in a form of a map. The inset result of fitting the monoexponetial decay to the integrated intensity of the whole PL spectrum. 
In Fig. 3 we present a series of spectra, each measured during the $5 \mathrm{~ns}$ time interval, in a full time range of up to $2 \mu \mathrm{s}$ after the laser pulse. As can be expected, the recombination of the direct excitons (when electron and hole are localized within the same quantum well) is very fast and its decay time is of about a nanosecond. Because of the spatial separation of electrons and holes, in case of indirect excitons, the decay time is much longer. The indirect recombination arises slowly (left part) and then decays over microseconds. Moreover, it can be observed that together with the luminescence decay the electric field in the structure continuously increases. A similar effect was observed under varying the excitation intensity in experiments with continuous wave excitation - the higher intensity of the incoming light results in lowering the electric field in the structure. For rough estimation of the characteristic timescale of the indirect luminescence, the monoexponential decay was fitted to the luminescence intensity against time, yielding the decay time of $136 \mathrm{~ns}$.

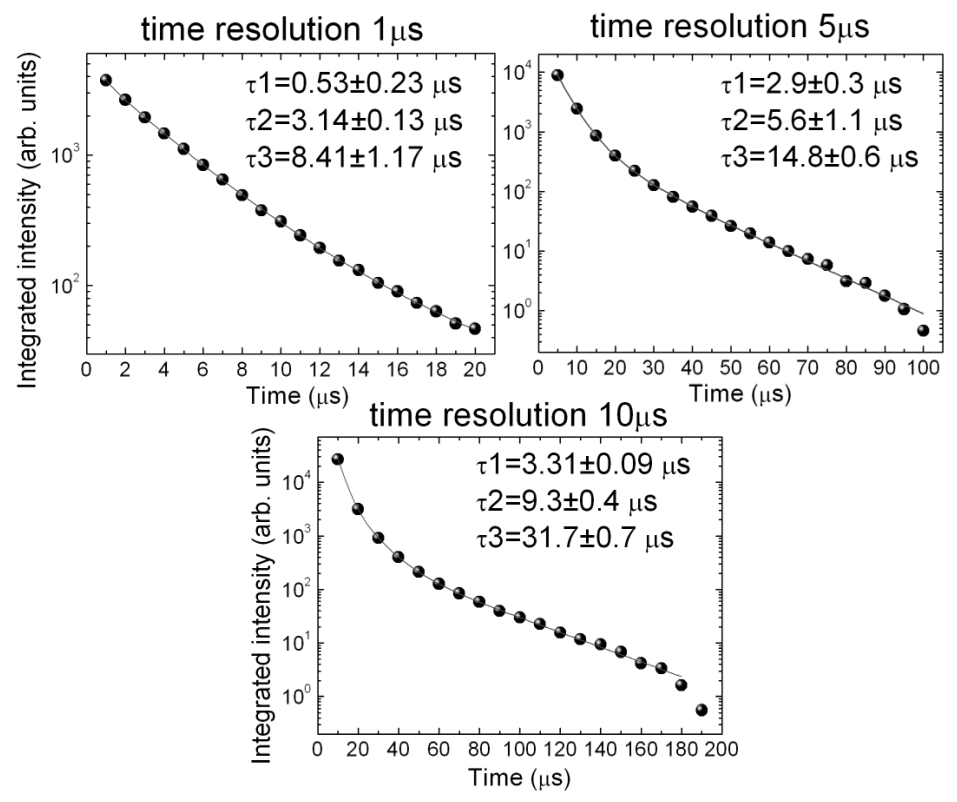

Fig. 4. Time evolution of the intensity measured for indirect transition with different time resolutions of $1 \mu \mathrm{s}$ (left), $5 \mu \mathrm{s}$ (middle), $10 \mu \mathrm{s}$ (right). Experimental data (symbols) are fitted with the third order exponential decay (see text).

In Fig. 4, the decays of the integrated intensity of the indirect luminescence are shown at different timescales: in time intervals up to 20, 100 and $200 \mu$ s with the resolution of 1,5 , and $20 \mu \mathrm{s}$. It can be seen that the monoexponential decay is rather poor approximation to reproduce the time evolution of the PL intensity in the whole time range [3]. The approach to fit the data shown in Fig. 4 with 


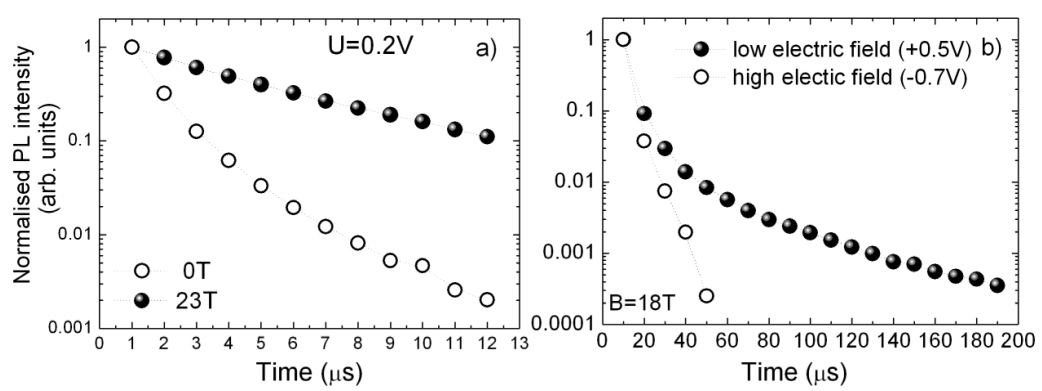

Fig. 5. Time dependence of the integrated intensity of indirect PL measured for: (a) constant electric and two different magnetic fields and (b) constant magnetic field and two different electric fields.

a sum of three exponential decays is shown with solid lines. This approach yields characteristic decay times from $0.5 \mathrm{~ns}$ for the PL evolution just after the laser pulse up to $30 \mu$ s for the PL decay long after the laser pulse.

Dynamics of indirect luminescence is found to be significantly influenced by the application of the magnetic field. As shown in Fig. 4, the application of magnetic fields notably slows down the decay of the indirect PL.

It was expected that the increase in the electric field leads to the increase in the spatial separation of carriers which in consequence may result in the observation of longer decay times. Such a result was presented on a similar structure in [4]. Surprisingly enough, it was found that for our structure, the increase in the electric field strength results in the decrease in the decay time (see Fig. 5b).

\section{Discussion}

To describe the observed kinetics of indirect recombination one has to take into account relaxation processes within the structure. After the pulsed excitation some carriers are captured immediately in the wells and recombine rapidly as direct excitons (see Fig. 3). Due to electric field present in the structure electrons and holes relax preferentially into different wells, and after a short period of fast direct recombination, indirect transition dominates. The non-monoexponential decay of the indirect luminescence seen for a wide time range can be understood taking into account localization processes which influence the relaxation and recombination in the structure. One has to consider different traps in the structure (ionized donors, acceptors, fluctuations, etc.) that can capture the carriers after the excitation pulse and later can release them leading to the long time decay of indirect recombination. When the electric field is higher, it is easier to release captured carriers from donors, acceptors and potential fluctuations. These carriers can drop into the quantum wells and recombine there. For the higher electric field the carriers are released faster by the traps which results in a decrease in luminescence decay time.

This reasoning can also explain the observed increase in the decay time upon raising the magnetic field strength. $B$-field influences the carriers' localization at 
the potential fluctuations. The cyclotron radii of electrons and holes decrease proportionally to $B^{-1 / 2}$ causing that the carriers are more effectively captured by the impurities and potential fluctuations, increasing the decay time.

Similar mechanism can influence recombination processes in double well structure when the magnetic field is applied. Electrons and holes are better localized at random positions in different quantum wells so the recombination probability decreases with the increase in magnetic field.

\section{Conclusions}

We have shown that long decay time of the indirect luminescence originates from the localization effects. These effects are likely to be avoided in samples dedicated for demonstration of the Bose-Einstein condensation of excitons in double quantum well structures.

\section{Acknowledgments}

This work was partially supported by research framework program MTKDCT-2005-029671.

\section{References}

[1] L.V. Butov, C.W. Lai, A.L. Ivanov, A.C. Gossard, D.S. Chemla, Nature 417, 47 (2002).

[2] D.W. Snoke, S. Denev, Y. Liu, L. Pfeiffer, K. West, Nature 418, 754 (2002).

[3] A. Morel, P. Lefebvre, S. Kalliakos, T. Taliercio, T. Bretagnon, B. Gil, Phys. Rev. $B$ 68, 045331 (2003).

[4] A. Aleksandrou, J.A. Kash, E.E. Mendez, M. Zachau, J.M. Hong, T. Fukuzawa, Y. Hase, Phys. Rev. B 42, 9225 (1990). 